entity in general rather than for the individual patient with the illness, e.g. 'the prognosis of schizophrenia depends on ...' rather than 'the good prognostic features in this patient are ...'

There are a number of elements which determine the prognosis and which you might bring in. You will often find a mixture of good and bad prognostic features which you will need to balance up in arriving at your final judgement.

You might consider the following:

(a) Known prognostic features of the illness as applied to this particular patient, e.g. affective 'colouring' in a schizophrenic illness, acute onset, etc.

(b) The course of the illness-if it is already chronic it is likely to remain so.

(c) Response to treatment in the past.

(d) Co-operation with treatment in the past and now.

(c) Premorbid adjustment.

(f) Social supports and influences.

(g) Motivation to improve.

(h) The availability of special treatment facilities.

It may be helpful to divide the prognosis into the short term and long term, e.g. the patient may have a good prognosis for recovery from the current episode but be at high risk for relapse in the future.

\section{Staff communication}

Dear Sir

Having read the account of the staff support system at Hill End Adolescent Unit (Bulletin, July 1982, 6, 117-19) I very much doubt whether the desired open communication can be fostered in such a culture. Staff meetings in which the use of first names is compulsory, in which statements are prohibited which are not ' $I$ ' statements, and in which there are rules which forbid conversation about absent colleagues and patients, strike me as being every bit as defensive, restricted in communication, and tyrannical as the hierarchical system which the authors purport to eschew.

The danger of such groups is that the members are forced into a pattern of pseudo-open communication in order to conform to rigid group norms; thus more is avoided than is dealt with, tension is greater, and there are repercussions elsewhere in the system.

Central Middlesex Hospital

Harold L. BeHR

London NW10

\section{Psychiatric experts and expertise-will the real expert please stand up?}

\section{Dear Sir}

The article by the barrister, Diana Brahams, on 'Psychiatric testimony-Who can give it and when?' (Bulletin, July 1982, 6, 121-22) raises a number of interesting points as to who is best qualified to give evidence on problems of mental and behavioural abnormality.

In the case cited by Mrs Brahams (Mackenny and Pinfold) I would accept that the qualifications of the psychologist (who was not allowed by the trial judge to give evidence) could be called into question, but I wonder how the matter might have been resolved if the psychologist involved had been a properly trained clinical psychologist -employed by the National Health Service-who had experience in diagnosis and treatment of mental disorder, and who was conversant with preparing reports for solicitors as well as in giving expert evidence in courts, be they at Magistrate, County or higher courts.

My own experience in legal matters indicates that clinical psychologists not only provide reports for solicitors, but that in many instances solicitors (as well as barristers) specifically request a psychologist's report in preference to or, in conjunction with a medical or psychiatric report. Moreover, examples of cases where psychologists are requested to attend Court to give evidence include Compensation (brain damage, psychological effects of personal injury); Matrimonial (access, custody, care proceedings); Juvenile and Adult Crime (burglary, damage to property, murder, rape, theft), not to mention acting in an advisory capacity to the legal profession.

I can recall an occasion (the first time I gave evidence) when the 'other side' in a compensation case objected to my report-and presumably me as well-being granted 'expert' status. The learned judge, after listening to counsel's objections, took a few moments before giving his decision on my report, namely-'Oh nonsense, put it with all the rest!' (i.e. the medical reports). Despite my nod of approval at the time, I later realized that His Lordship had poured equal scorn on both the so called medical and psychological expertise. A fuller account of this incident has been reported elsewhere (Kaufman, 1980).

A few months ago I was asked by another judge if I was qualified to give an opinion on a man's state of mind concerning whether or not he was suffering from an 'abnormality of mind' at the time he took money belonging to his firm, because, after all, I was 'not a psychiatrist'. My reply was that not only did I think I was qualified to offer my opinion, but that in my experience as well as that of many of my colleagues, we are often referred cases (by psychiatrists) for our diagnostic assistance. I also pointed out that in one area of clinical psychology specialization, the task is one of deciding if an abnormality in behaviour or deterioration in intellectual function is due to an organic as opposed to a non-organic cause and, in some instances, to help pinpoint the site of the lesion in diagnoses of cerebral deficit.

These explanations appeared to satisfy the learned judge and no more was said of my qualifications to give evidence as a clinical psychologist or, to give evidence on the matters in question, even by the very thorough opposing barrister appearing for the prosecution.

I can cite other instances where I have been asked to 
comment freely (including commenting on certain aspects of medical evidence) on issues where the professional boundaries are not particularly clear cut (e.g. diagnosis of early dementia) and where individual skill and experience is a key factor as opposed to what titles or labels one happens to have before or after one's name. Indeed, in some instances relating to brain function, I would have no hesitation in advising a solicitor or barrister to challenge a medical person's competence to offer an expert opinion in a Court of Law.

It seems ironical to non-medical professionals who have written papers or given lectures on specific topics (for doctors) then to be challenged, because they are not doctors, on their right to practice as experts within the legal framework. Hopefully, these are matters that will come right in time as courts become more and more familiar with the varying (and overlapping skills) of various professional groups.

One particularly difficult problem-as Mrs Brahams rightly reminds us - is that of bringing to court 'a whole constellation of expert witnesses' whenever there is 'a whisper of abnormality'. Although I would agree that there is certainly a danger of too many experts (or perhaps too many experts from too many fields) getting in 'on the act', there are almost certainly greater risks entailed when issues that are by no means clear cut are allowed to become the exclusive province of one discipline-as the ghosts of the Ripper trial will continue to remind us for some time to come.

\section{Children's Hospital}

Western Bank, Sheffield

REFERENCE

KAufman, A. (1980) A closed shop on healing? World Medicine, 3 May, 43-44.

\section{Planning registrar and senior registrar training in mental handicap}

DeAR SIR

I wish to congratulate Dr Spencer for his outline of training activities for registrars and senior registrars in mental handicap (Bulletin, May 1982, 6, 82). I would, however, like to state that the training standards of registrars and senior registrars in mental handicap should be similar to those of trainees in adult psychiatry. The sad fact is that this is not the case.

Most registrar and senior registrar posts are occupied by foreigners on an indefinite locum basis. There is very little exchange of knowledge between consultant and trainee, i.e., no teaching or very little. No admissions, no discharges, no journal clubs, no case presentations. Library facilities are inadequate, if not ancient. The work is basically that of a GP looking after the general health needs of his mentally-handicapped patients. In institutions which are 'progressive' the registrar/senior registrar attends case presentations at the postgraduate centre of a psychiatric complex.

Training in mental handicap must change and reach the standards of that in adult psychiatry.

R. ARmah-KwANTreng

Botleys Park Hospital

Chertsey, Surrey

\section{Trainees' views of approval visits}

\section{DeAr Sir}

I think Philip Thomas (Bulletin, July 1982, 6, 124-25) may have made some unwarranted assumptions as to why trainees from teaching hospitals were much less satisfied with the accuracy of the reports of Approval Panels compared with trainees from peripheral hospitals. It would be of interest to look at the relationship between the final result of the Approval visit (A, P or U) and the satisfaction or dissatisfaction expressed by the trainees. It would seem to me that there might be a 'halo effect' in that trainees would be more inclined to be satisfied with the approval team providing the highest grade of Approval and vice versa.

In this Region the peripheral hospitals in general have done rather better than several of the teaching hospitals with regard to their category of Approval. The reason for satisfaction amongst trainees in these peripheral hospitals may be their satisfaction with the outcome of the visit, or satisfaction with their hospital which has perhaps earned a high Approval category. To make the assumption that trainees in peripheral hospitals are demoralized and faced with inertia or lack of interest from their senior colleagues seems to be unwarranted if the true situation is as described above.

ROGER W. WHITELEY

Central Hospital

Warwick 